\title{
Design \& Implementation of Auto-Stabilizing Bike
}

\author{
Bhagirathi Bai V*, Aishwarya P, Akhil G, Kevin T, Shardul \\ Mechatronics Engineering, Acharya Institute of Technology, Bangalore
}

DOI: https://doi.org/10.21467/proceedings.1.51

* Corresponding author email: bhagirathistelkar@gmail.com

\begin{abstract}
This paper explores self balancing bicycle design and implementation. The automatic balancing bicycle will utilize a system capable of keeping itself upright in the event of any imbalance caused during motion. For the purpose of balancing \& propelling a motor $\&$ power system will provide the required power \& torque. In addition to being able to balance during motion, it will be capable of stopping without losing its balance. A sensor will be used to detect tilt angle \& the accelerometer-gyroscopic sensor will then strive to maintain the vertical position based on it.

Index Terms- Accelerometer, Balancing bicycle, Balancing control, Gyroscope, Tilt angle, Self-balance
\end{abstract}

\section{INTRODUCTION}

Cycling is used for many purposes such as exercise, transportation, recreation due to it being a low impact form of exercise training co-ordination, stamina, strength, balance, etc. The task of learning to ride a bicycle however may seem daunting to children, injured people, elderly, etc. A system providing balance assistance would then be beneficial to these group of individuals. Such a self-self-balancing bicycle will reduce the learning period while enhancing the experience of riding a bicycle. The basic idea is to have a system capable of applying appropriate torque required to keep the bicycle in vertical position while taking into account factors such as geometry, mass distribution, speed of the bike, frame, etc. The idea to build a self-balancing bicycle was originally adopted from Honda's "ride assist-e" technology that used hydraulics to constantly wobble the front wheel and vary the wheel base to maintain its balance equilibrium, with the help of sensors attached to the riders gloves and helmet

\subsection{Objective}

Designing and implementing an autonomous self balancing bicycle using an accelerometer gyroscopic sensor, motor \& a power source. The design must be stable for average range of weight with allowances for slight deviations in tilt and position.

\subsection{Problem definition \& solution}

Initially learning to ride a bicycle is not a simple task, one may have to practice for number of hours \& use training wheels to gain enough confidence to ride it smoothly. This is especially

(C) 2018 Copyright held by the author(s). Published by AIJR Publisher in Proceedings of the $3^{\text {rd }}$ National Conference on Image Processing, Computing, Communication, Networking and Data Analytics (NCICCNDA 2018), April 28, 2018.

This is an open access article under Creative Commons Attribution-NonCommercial 4.0 International (CC BY-NC 4.0) license, which permits any non-commercial use, distribution, adaptation, and reproduction in any medium, as long as the original work is properly cited. ISBN: 978-81-936820-0-5 
Design \& Implementation of Auto-Stabilizing Bike

true for young children, people with disabilities, injured \& elderly. Thus, a system which will assist in teaching $\&$ boosting confidence at the same time would be a great boon to these individuals. A self-balancing bicycle would benefit even bike trailing enthusiasts when injured or overcome with fatigue. Such a system would allow the rider to exert no effort balancing the bike.

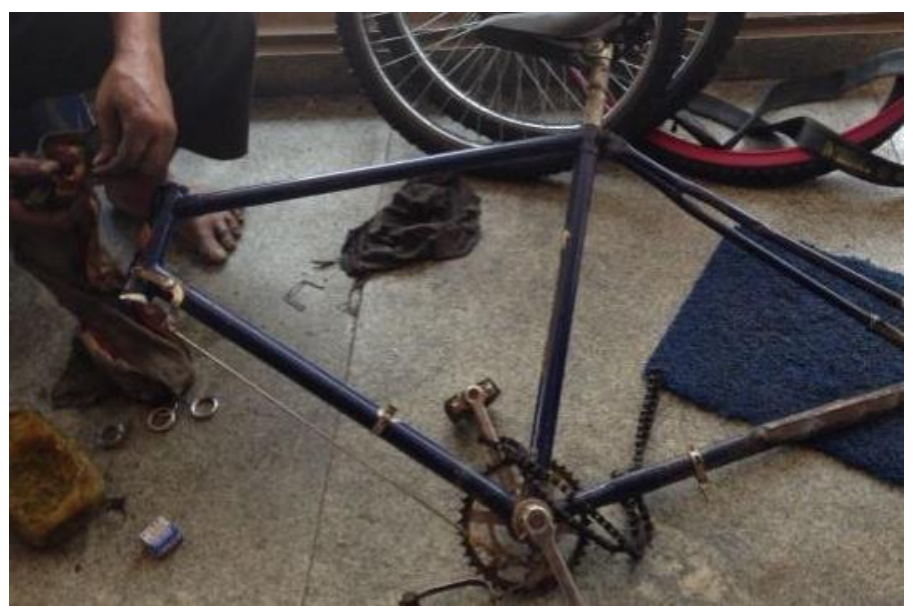

\section{Methodology}

The methods to achieve balancing are of several types- flywheel design, gyroscopic effect, steering control, etc. Out of these methods the one based on the gyroscopic effect, the torque provided would be sufficient and the response time is satisfactory. Using an integrated gyroscope \& accelerometer, the tilt angle information provided would be more accurate than if the sensors were used separately.

\section{Stages:}

Stage 1: Basic idea \& literature survey

Stage 2: Research on hardware \& software requirements \& compatibility

Stage 3: Design considerations to minimize drawbacks

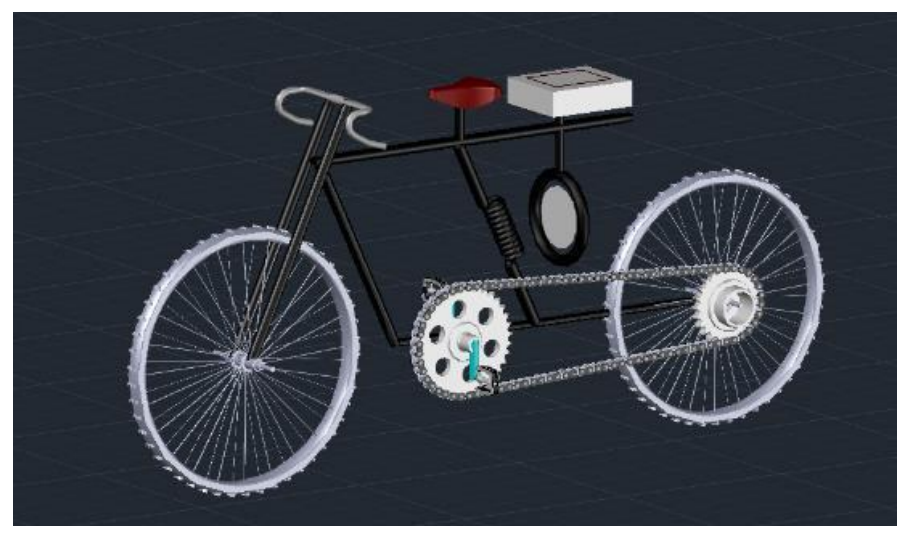




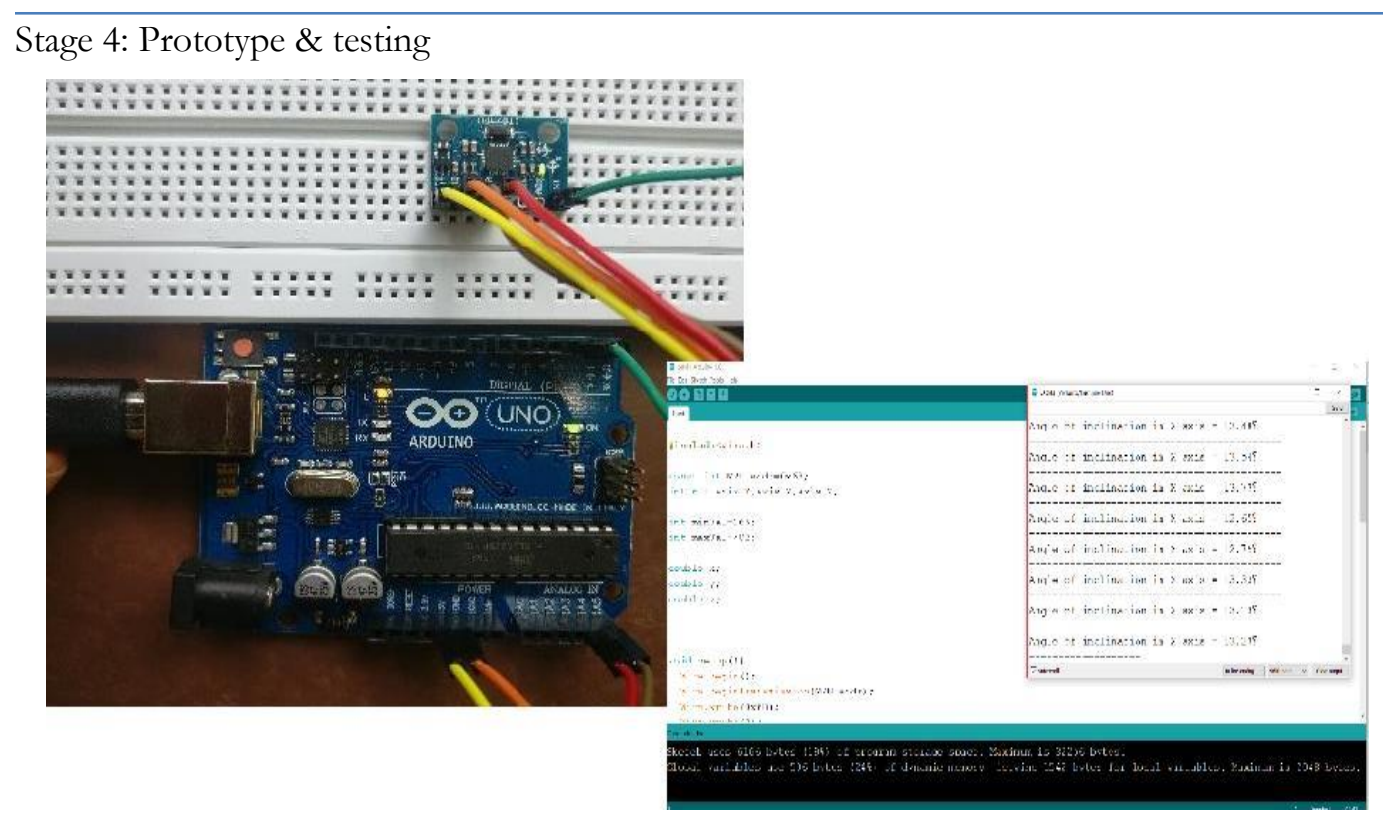

Stage 5: Evaluation \& documentation

\section{Hardware:}

Arduino uno, Motor, motor driver, power source, MPU6050 sensor, etc

\section{FLOWCHART}

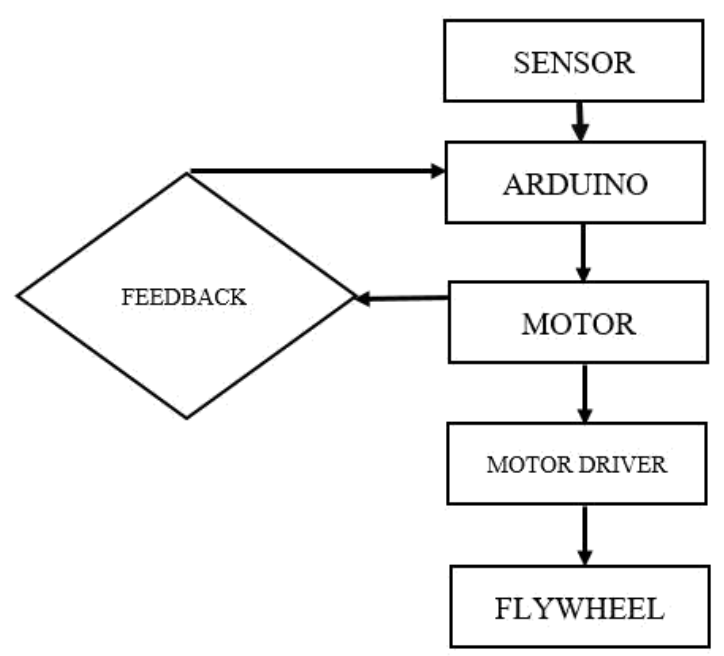

\section{RESULT AND ANALYSIS}

We were able to find the tilt angle using MPU6050 sensor (accelerometer and gyroscopic sensor), this sensor is useful in finding the angle of tilt created by the bicycle during motion. With the obtained output we were able to calculate the time available to change the direction of rotation of the motor. With this information we were able to calculate the torque required

Proceedings of the $3^{\text {rd }}$ National Conference on Image Processing, Computing, Communication, Networking and Data Analytics (NCICCNDA 2018) 
to run the flywheel in the opposite direction so as to maintain its equilibrium. The various tilt

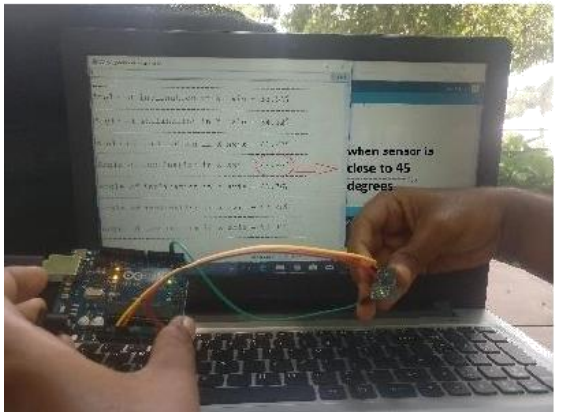

angles are as shown below

a.When the sensor is at 45 degrees.

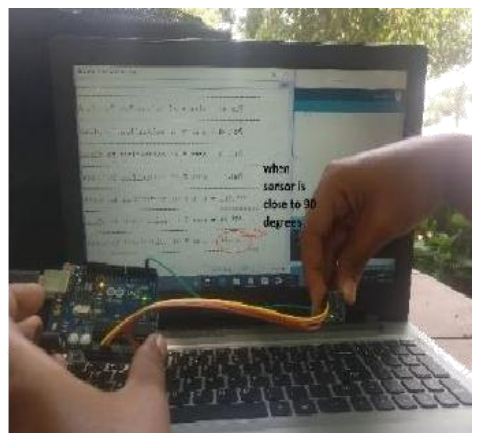

b. When the sensor is at 90 degrees.

c.When the sensor is at zero degrees

\section{CONCLUSIONS}

The main function of a self-balancing bicycle would be balance assist. Thus it would be possible to navigate over rough terrain, slippery roads, etc with ease. It would also benefit novice cyclist. It would be possible for a cyclist who may be injured or fatigued to be a able to balance the bicycle because of this system. The maximum allowed range of cyclist weight for the current design needs testing. Also further experimentation for bicycle working under

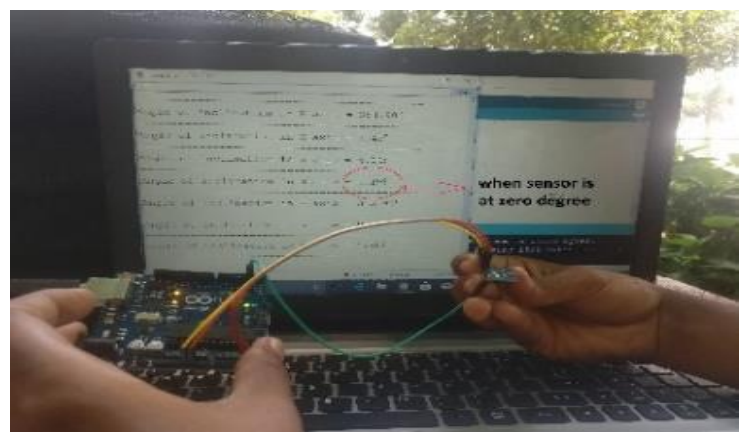

slippery conditions is needed. Since the MPU6050 sensor integrates accelerometer as well as gyroscope the output will be more accurate and less susceptible to interferences.

\section{REFERENCES}

[1] S.K. Gupta and V. Gulhane, "Pose estimation algorithm implication for bicycle using gyroscope \& accelerometer," Design approach, vol. 4, 2014.

[2] J. He and M. Zhao, "Control system design of self-balanced bicycles by control moment gyroscope," in Proceedings of the 2015 Chinese Intelligent Automation Conference, pp. 205-214, 201

[3] K. Kanjanawanishkul, "LQR and MPC controller design and comparison for a stationary self-balancing bicycle robot with a reaction wheel," Kybernetika, pp. 173-191, 2015.

[4] N. Aphiratsakun and K. Techakittiroj, "Autonomous AU bicycle: Self-balancing and tracking control (AUSB2)," 2013 IEEE International Conference on Robotics and Biomimetics, 2013. 